\title{
Protective Effect of Tetrahydrocurcumin against Cisplatin-Induced Renal Damage: In Vitro and In Vivo Studies
}

Authors

Affiliations
Kyung II Song ${ }^{1 *}$, Jun Yeon Park ${ }^{2 *}$, Seungyong Lee ${ }^{3,4}$, Dahae Lee ${ }^{2,4}$, Hyuk-Jai Jang ${ }^{5}$, Su-Nam Kim ${ }^{3}$, Hyeonseok Ko ${ }^{6}$, Hyun Young Kim ${ }^{4}$, Jae Wook Lee ${ }^{3}$, Gwi Seo Hwang ${ }^{2}$, Ki Sung Kang ${ }^{2}$, Noriko Yamabe ${ }^{2}$

The affiliations are listed at the end of the article

\author{
Key words \\ - cisplatin \\ - nephrotoxicity \\ - tetrahydrocurcumin \\ - oxidative renal damage \\ - cOX-2
}

\begin{abstract}
$\nabla$

The adverse effects of anticancer drugs can prompt patients to end their treatment despite the efficacy. Cisplatin is a platinum-based molecule widely used to treat various forms of cancer, but frequent and long-term use of cisplatin is limited due to severe nephrotoxicity. In the present study, we investigated the protective effect and mechanism of tetrahydrocurcumin on cisplatininduced kidney damage, oxidative stress, and inflammation to evaluate its possible use in renal damage. Cisplatin-induced LLC-PK1 renal cell damage was significantly reduced by tetrahydrocurcumin treatment. Additionally, the protective effect of tetrahydrocurcumin on cisplatin-induced oxidative renal damage was investigated
\end{abstract}

\section{Introduction}

DOI http://dx.doi.org/

10.1055/s-0035-1545696

Published online February 26, 2015

Planta Med 2015; 81: 286-291

(c) Georg Thieme Verlag KG

Stuttgart · New York .

ISSN 0032-0943

\section{Correspondence}

Noriko Yamabe

College of Korean Medicine

Gachon University

1342 SeongnamDaero

Seongnam 461-701

Korea

Phone: +82317505421

Fax: +82317505416

norikoy@gachon.ac.kr

Correspondence

\section{Ki Sung Kang}

College of Korean Medicine

Gachon University

1342 SeongnamDaero

Seongnam 461-701

Korea

Phone: + 82317505402

Fax: +82317505416

kkang@gachon.ac.kr

\section{$\nabla$}

The usual treatments for cancer are surgery, chemotherapy, radiation, or a combination of these methods $[1,2]$. Chemotherapy is the use of anticancer drugs to treat cancerous cells. It has been used for many years, and is one of the most common treatments for cancer $[3,4]$. Anticancer drugs interfere with the growth of tumor cells, eventually causing their death. However, they may also affect the growth of normal cells, causing many adverse effects, some of which may be serious [5]. Cisplatin is a platinum-based anticancer drug widely used to treat various forms of cancer in humans [6,7]. However, this treatment has some major limitations for use. Within an hour following injection, some patients suffer from side effects that include perception and hearing disorders, tinnitus, and inner ear dysfunctions that

\footnotetext{
* These two authors contributed equally to the work described in this study.
}

in rats. Tetrahydrocurcumin was orally administered every day at a dose of $80 \mathrm{mg} / \mathrm{kg}$ body weight for ten days, and a single dose of cisplatin was administered intraperitoneally $(7.5 \mathrm{mg} / \mathrm{kg}$ body weight) in $0.9 \%$ saline on day four. The creatinine clearance levels, which were markers of renal dysfunction, in cisplatin-treated rats were recovered nearly back to normal levels after administration of tetrahydrocurcumin. Moreover, tetrahydrocurcumin exhibited protective effects against cisplatin-induced oxidative renal damage in rats by inhibiting cyclooxygenase- 2 and caspase- 3 activation. These results collectively provide therapeutic evidence that tetrahydrocurcumin ameliorates renal damage by regulating inflammation and apoptosis.

cause dizziness $[8,9]$. In addition, frequent and long-term use of cisplatin is restricted because of severe nephrotoxicity $[10,11]$. These side effects can prompt patients to stop treatment despite its efficacy. Therefore, a better understanding of these side effects is crucial for the continued clinical use of cisplatin.

We have investigated naturally occurring antioxidants that can protect the kidney from cisplatininduced damage [12-14]. Many researchers have shown the involvement of oxidative stress in cisplatin-induced nephrotoxicity $[15,16]$. Cisplatin induces the generation of various reactive oxygen species (ROS) by inactivating the cellular antioxidant system, disrupting the mitochondrial respiratory chain, or interacting with microsomal cytochrome P450 [17,18]. Natural antioxidants provide beneficial effects against cisplatin chemotherapy, not only by protecting the kidney from cisplatin-induced oxidative damages, but also by inducing cancer cell death. For example, we have reported that antioxidants from Panax ginseng exhibited both renoprotective and anticancer ef- 
A<smiles>COc1cc(CCC(=O)CC(=O)CCc2ccc(O)c(OC)c2)ccc1O</smiles>

B

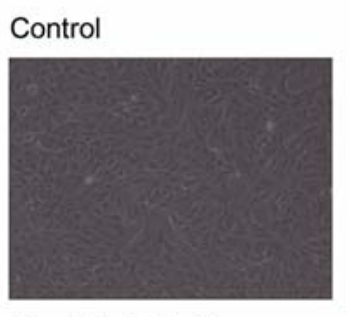

Cisplatin $(25 \mu \mathrm{M})$

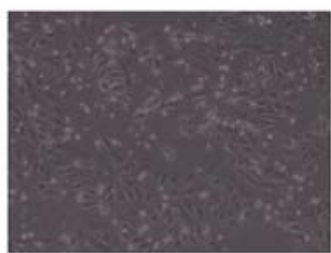

Cisplatin $(25 \mu \mathrm{M})+$ THC $(25 \mu \mathrm{M})$

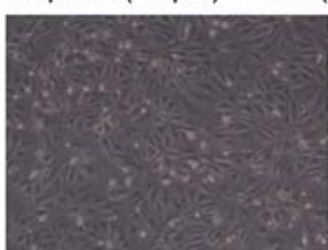

C

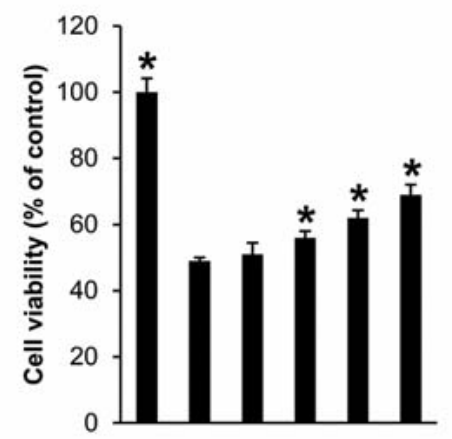

Cisplatin $(\mu \mathrm{M}) \quad 0 \quad 25 \quad 25 \quad 25 \quad 25 \quad 25$

$\operatorname{THC}(\mu \mathrm{M}) \quad 0 \quad 0 \quad 12.52550100$

D

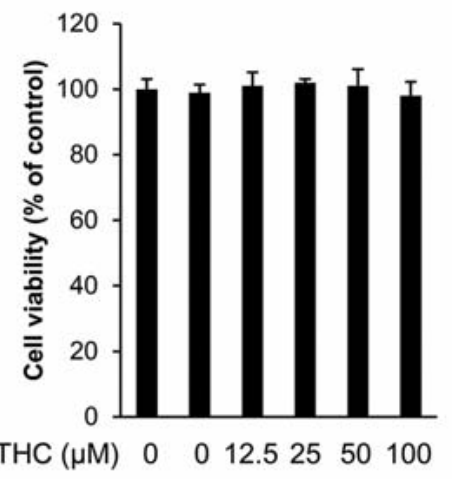

Fig. 1 Effect of tetrahydrocurcumin on cisplatininduced nephrotoxicity in LLC-PK1 cells. A Chemical structure of THC. B Representative microscopic images showing the protective effect of THC against cisplatin-induced nephrotoxicity in cells. C Dose-dependent protective effect of THC against cisplatin-induced nephrotoxicity in cells. D No cytotoxic effects of THC at the same doses, which was effectively protected from cisplatin-induced cell damage. $\mathrm{P}<0.05$ compared to the cisplatin-treated control value. (Color figure available online only.) fects, providing evidence for its use as a chemotherapeutic adjuvant $[19,20]$.

Curcumin is an active ingredient from the plant Curcuma longa $\mathrm{L}$. (Zingiberaceae), which has various pharmacological effects, including anti-inflammatory, antioxidant, and anticarcinogenic activities [21-23]. Tetrahydrocurcumin (THC) is an active metabolite of curcumin found in the gastrointestinal tract and a reduced analog of curcumin with phenolic and $\beta$-diketo moieties ( Fig. 1 A). THC possesses stronger antioxidant activity than other curcuminoids, including curcumin, demethoxycurcumin, and bisdemethoxycurcumin $[24,25]$. However, little is known about the effects of THC on cisplatin-induced nephrotoxicity. Therefore, the in vitro and in vivo effects and molecular mechanisms of THC on cisplatin-induced nephrotoxicity were investigated in this study.

\section{Results and Discussion}

$\nabla$

Recent literature indicates that ROS play critical roles in the development and progression of kidney damage [26-29]. Antioxidant treatment can prevent oxidative damage and might delay the progression of kidney disease. Therefore, the use of antioxidant agents as inhibitors against oxidative stress may be considered an important therapeutic approach for kidney disease. In the present study, we have investigated the protective effects and mechanism of THC on cisplatin-induced kidney damage, oxidative stress, and inflammation to evaluate its possible use in renal damage.

The protective effect of THC against cisplatin-induced oxidative damage was tested using LLC-PK1 cells, which are renal tubular cells that are the most vulnerable renal tissue to oxidative stress [26]. Fig. 1 B shows the morphological changes of cisplatintreated LLC-PK1 cells that were also treated with THC. As shown in $\odot$ Fig. $1 \mathrm{C}, 25 \mu \mathrm{M}$ cisplatin treatment significantly decreased cell viability to about $50 \%$ of that of untreated control cells. In contrast, pretreatment with THC markedly restored cell viability in a dose-dependent manner. However, THC demonstrated no cytotoxic effects at the same doses, which was effectively protected from cisplatin-induced cell damage ( Fig. 1D). Then, we further examined the effect of THC on cisplatin-induced oxidative renal damage in rats.

The body weight gains of rats after cisplatin and cisplatin + THC treatments were markedly reduced compared to normal rats 


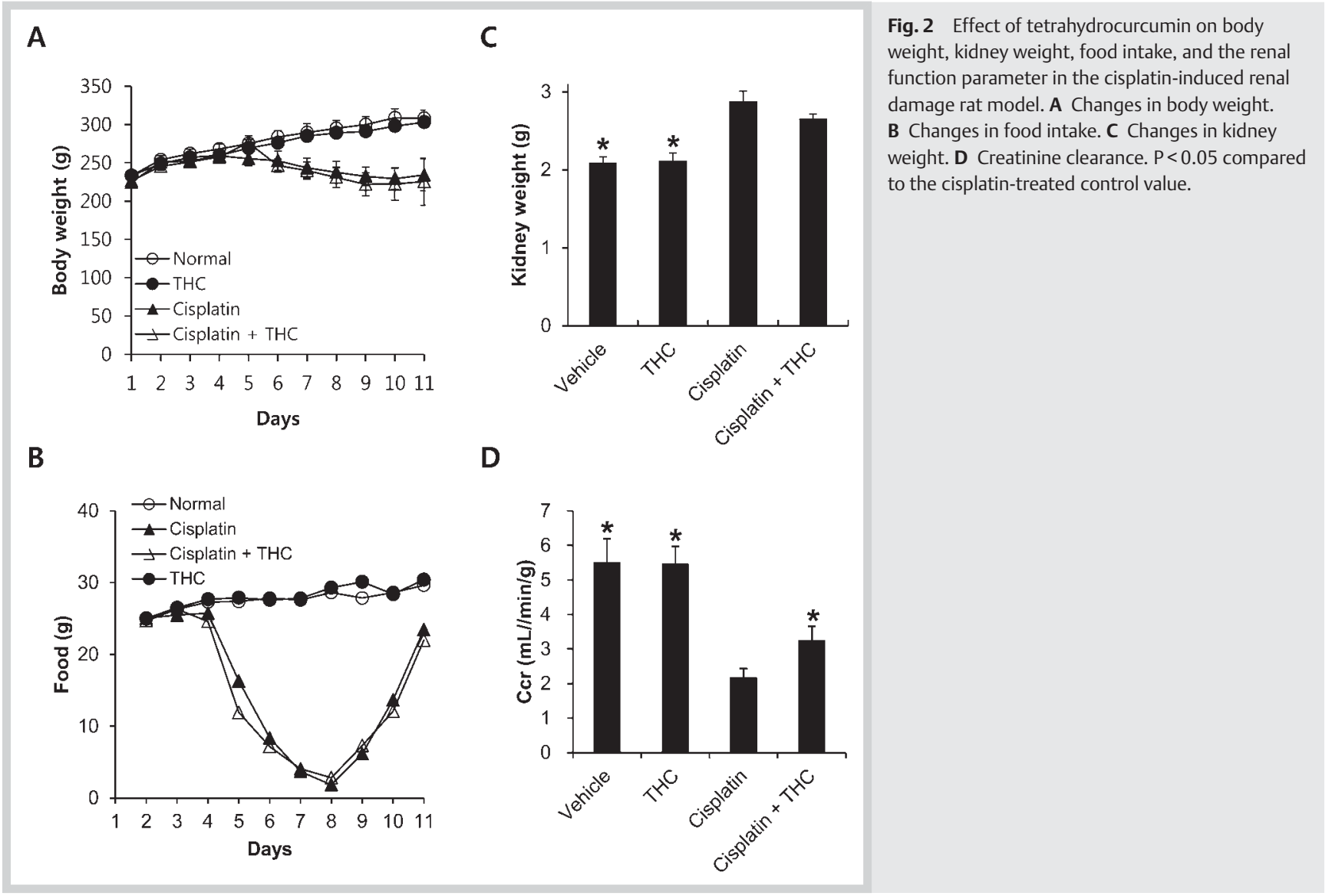

(๑ Fig. 2A). Similarly, food intake amounts were slightly lowered after cisplatin treatments and gradually recovered in the vehicletreated groups ( $\bullet$ Fig. 2B). The sharp decrease in the food intake was observed on day four in cisplatin and cisplatin + THC treated groups. These results are in accordance with earlier reports that noted a decrease in body weight gain after cisplatin injection [30]. Cisplatin intoxication resulted in a significant body weight reduction and kidney weight increase. However, these changes were not significantly ameliorated by THC treatment ( $\bullet$ Fig. $2 \mathrm{C}$ ). Regarding renal functional parameters, cisplatin-injected rats exhibited decreased creatinine clearance levels compared to those of the vehicle-treated group. The reduced creatinine clearance level of cisplatin-treated rats was significantly recovered by cotreatment with THC ( Fig. 2D).

- Fig. 3 A shows the effect of THC on organic cation transporter-2, cyclooxygenase-2, and procaspase-3 protein expression in cisplatin-treated rat kidneys. OCT-2 is a renal uptake transporter that plays a key role in disposition and the renal clearance of drugs and endogenous compounds [31]. OCT-2 protein expression in kidney tissue was slightly increased after cisplatin treatment and was decreased by THC treatment, but the differences were not statistically significant ( Fig. 3B). COX-2 is an inducible component of the prostaglandin synthesis cascade and is inducible in normal cells by many cytokines, mitogens, and proinflammatory factors [32]. Recent studies have demonstrated that COX2 is highly expressed in response to inflammation in kidney tissue. Therefore, it may be intimately involved in prostaglandindependent renal inflammatory processes [33]. Thus, the development of COX-2 inhibitors serves as a paradigm for molecularly targeted renoprotective agents. Caspase- 3 is potentially the most important effector enzyme in apoptosis, providing a common pathway to both death receptor- and mitochondria-dependent apoptotic mechanisms $[34,35]$. COX-2 and procaspase-3 protein expression levels were significantly increased after cisplatin-injection, but cisplatin co-treatment with THC resulted in almost complete renoprotection. These results imply that THC may alleviate oxidative stress by preventing caspase- 3 activation and related inflammation in the kidney.

PAS staining was performed on renal sections to measure tubular damage. As shown in the representative pictures of renal sections, severe tubulointerstitial injuries, including tubular epithelial cell detachments, cystic dilatation of tubules, and inflammatory cell infiltration, occurred in the kidneys of cisplatin-treated animals ( Fig. 4). However, the increased tubular damage in cisplatin-treated rats was reduced by co-treatments with THC ( Fig. 4). Therefore, THC was effective in alleviating cisplatin-induced tubulointerstitial injuries.

In summary, kidney cell damage induced by cisplatin was significantly inhibited by treatment with THC. In addition, the renal dysfunction of cisplatin-treated rats was markedly ameliorated by THC extract administration. The renoprotective effect of THC was associated with the caspase-dependent anti-inflammatory pathway. Taken together, these results demonstrate that THC exerted a renoprotective effect in cisplatin-treated rats and, therefore, its use can be considered to prevent kidney damage during or after chemotherapy. 


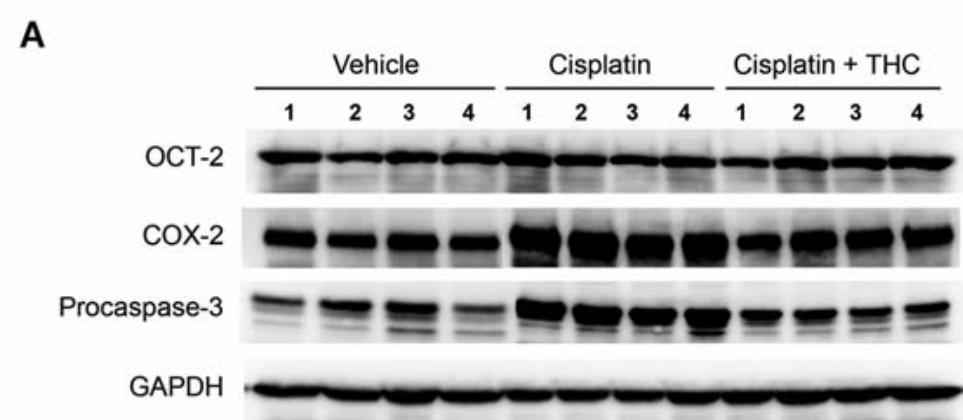

B
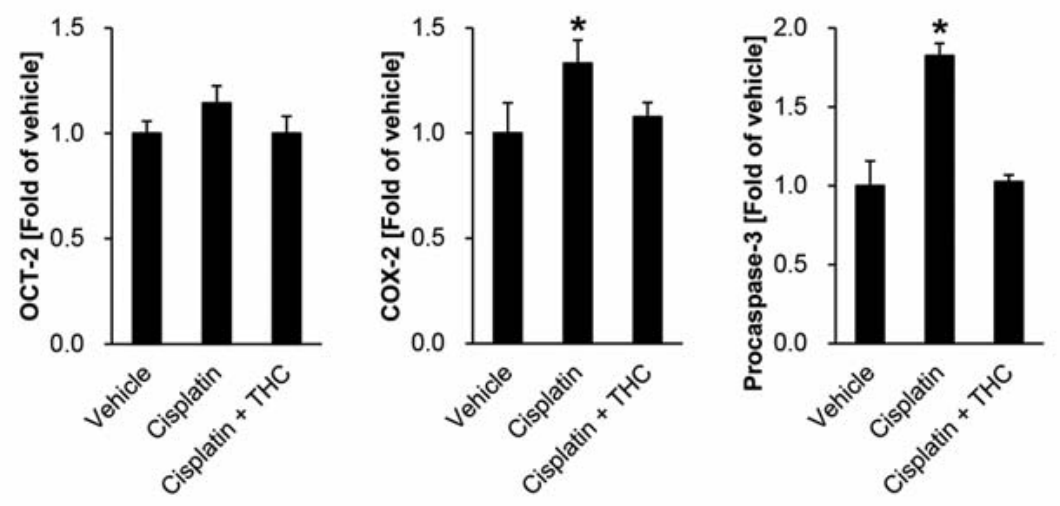

Fig. 3 Effect of tetrahydrocurcumin on protein expression levels of organic cation transporter-2, cyclooxygenase-2, and procaspase- 3 of the rat kidney. A OCT-2 (60 kDa), COX-2 (72 kDa), and cleaved caspase-3 $(17,19 \mathrm{kDa})$ protein expressions evaluated by Western blot analysis. GAPDH (37 kDa) was used as an internal control. B Quantitative data for the OCT-2, COX-2, and cleaved caspase-3 Western blot analysis. Western blot assays were done in triplicate for each protein and were repeated at least three times. $\mathrm{P}<0.05$ compared to the cisplatin-treated control value.
A

B

\section{Control}

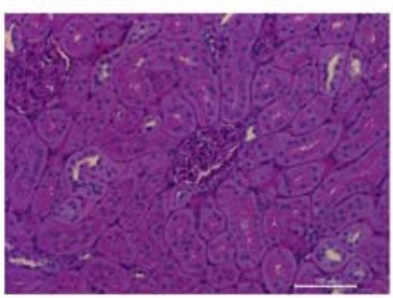

Cisplatin

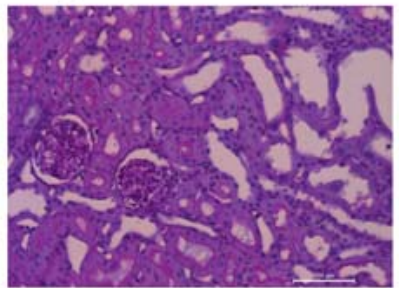

Cisplatin + THC

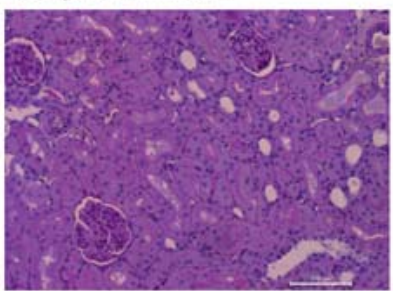

Fig. 4 Effect of THC on the cisplatin-induced tubular damage in renal cortex tissues. A PAS staining of a representative renal section. B Quantitative data for the tubular damage of PAS staining. Original magnification of $\mathbf{A}$ is $\times 100$. $P<0.05$ compared to the cisplatin-treated control value. (Color figure available online only.) 


\section{Materials and Methods}

$\nabla$

\section{Chemicals and reagents}

THC and cisplatin (purity 98-100\%) were purchased from Sigma. A stock solution of chemicals for cell-based assays was prepared in $100 \%$ DMSO and stored at $-20^{\circ} \mathrm{C}$ until use. When required, the stock solution was diluted with cell culture media to the appropriate concentration. The final concentrations of DMSO in the culture media were adjusted to less than $0.5 \%(\mathrm{v} / \mathrm{v})$, which exhibits no toxic effect for cells. Dulbecco's modified Eagle's medium (DMEM) was purchased from Cellgro and FBS was from Invitrogen. OCT-2, COX-2, cleaved caspase-3, GAPDH, and horseradish peroxidase (HRP) conjugated anti-rabbit antibodies were purchased from Cell Signaling and EZ-Cytox reagent was from Daeil Lab Service.

\section{Renoprotective effect against cisplatin-induced damage in kidney cells}

The renoprotective effect against oxidative renal damage was evaluated using LLC-PK1 cells [26]. LLC-PK1 (pig kidney epithelium, CL-101) cells were purchased from ATCC and cultured in DMEM supplemented with $10 \% \mathrm{FBS}, 1 \%$ penicillin/streptomycin, and $4 \mathrm{mM}$ L-glutamine at $37^{\circ} \mathrm{C}$ with $5 \% \mathrm{CO}_{2}$ in the air. The cells were seeded in 96-well culture plates at $1 \times 10^{4}$ cells per well and allowed to adhere for $2 \mathrm{~h}$. Thereafter, the test sample and/or radical donor, $25 \mu \mathrm{M}$ cisplatin, were added to culture medium. Twenty-four hours later, the medium containing the test sample and/or radical donor was removed. The cells were incubated with serum free medium $(90 \mu \mathrm{L} /$ well $)$ and Ez-Cytox reagent $(10 \mu \mathrm{L} /$ well) at $37^{\circ} \mathrm{C}$ for $2 \mathrm{~h}$. Cell viability was measured by absorbance at $450 \mathrm{~nm}$ using a microplate reader.

\section{Renoprotective effect against cisplatin-induced oxidative damage in rats}

All procedures involving the use of live animals as described in this study were approved in May 2014 by the Institutional Animal Care and Use Committee of the Gachon University (approval number GIACUC-R2 014002) and strictly followed the NIH guidelines for the humane treatment of animals. Male Wistar rats weighing 140-160 g were used for evaluating the protection of THC against cisplatin-induced nephrotoxicity. The rats were housed with a temperature of $23 \pm 2{ }^{\circ} \mathrm{C}$ and $55 \pm 5 \%$ humidity [conditions with a standard light ( $12 \mathrm{~h}$ light/dark)]. The rats were given free access to water and a normal diet containing $10 \mathrm{kcal} \%$ fat for a period of one week after their arrival and were then divided into four groups based on their body weight and assigned to the vehicle, THC, cisplatin, or cisplatin + THC.

Group 1: vehicle $(n=4)$, received water (no sample treatment); group 2: THC $(\mathrm{n}=4)$, treated with THC $(80 \mathrm{mg} / \mathrm{kg})$ in aqueous solution orally for ten days; group 3: cisplatin $(n=4)$ received water (no sample treatment); group 4: cisplatin + THC $(n=4)$, treated with THC $(80 \mathrm{mg} / \mathrm{kg})$ in aqueous solution orally for ten days.

THC was orally administered every day at a dose of $80 \mathrm{mg} / \mathrm{kg}$ body weight, while vehicle-treated rats were orally given water. The $80 \mathrm{mg} / \mathrm{kg}$ of THC dosage was chosen according to the literature [25]. After four days, rats in two groups (cisplatin and cisplatin + THC) were administered a single dose of cisplatin intraperitoneally $(7.5 \mathrm{mg} / \mathrm{kg}$ body weight) in $0.9 \%$ saline. Animals in the vehicle group received an equivalent amount of normal saline for ten days. The rats were sacrificed six days after cisplatin administration under light ether anesthesia. Twenty-four hour urine samples were collected using a metabolic cage. Blood sam- ples were collected from the abdominal aorta, and the kidneys were removed. All the preparations and analyses of various parameters were performed simultaneously under similar experimental conditions to avoid any day-to-day variations. During the experimental period, their body weights were measured daily.

\section{Plasma biomarker analyses}

Blood samples were collected in tubes containing 0.18 M EDTA and centrifuged at $5000 \mathrm{rpm}$ for $5 \mathrm{~min}$ at $4^{\circ} \mathrm{C}$. After centrifugation, the plasma was separated for the estimation of creatinine. Creatinine levels were determined by a rate-blanked kinetic Jaffe method. Creatinine clearance was calculated on the basis of the urinary $\mathrm{Cr}$, serum $\mathrm{Cr}$, urine volume, and body weight using the following equation:

Creatinine clearance $(\mathrm{mL} / \mathrm{min} / \mathrm{g}$ body weight $)=$ [urinary $\mathrm{Cr}(\mathrm{mg} /$ $\mathrm{dL}) \times$ urine volume $(\mathrm{mL}) /$ serum $\mathrm{Cr}(\mathrm{mg} / \mathrm{dL})] \times[1000 /$ body weight $(\mathrm{g})] \times[1 / 1440(\mathrm{~min})]$

\section{Histological analysis of kidney}

Kidney samples were fixed in $10 \%$ buffered formalin phosphate, dehydrated, embedded in paraffin, sectioned in $3 \mu \mathrm{m}$ thickness, and stained with periodic acid-Schiff (PAS) reagent (Fisher Scientific) for histological examination. Tubular damage in PAS-stained sections was examined under a microscope and scored based on the percentage of cortical tubules showing epithelial necrosis: 0 , normal; $1,1-25 \%$; 2, 26-50\%; 3, 51-75\%; 4, > 76\%. Tubular necrosis was defined as the loss of the proximal tubular brush border, blebbing of apical membranes, tubular epithelial cell detachment from the basement membrane, or intraluminal aggregation of cells and proteins, as described previously [36].

\section{Preparation of whole-cell extracts from tissue}

Whole-cell extracts from the kidney tissue were prepared according to the manufacturer's (Cell Signaling) instructions using RIPA buffer supplemented with a $1 \times$ protease inhibitor cocktail and $1 \mathrm{mM}$ phenylmethylsulfonyl fluoride (PMSF, Sigma).

\section{Western blot analysis}

Proteins (whole-cell extracts, $30 \mu \mathrm{g} / \mathrm{lane}$; nuclear extracts, $10 \mu \mathrm{g} /$ lane; cytosolic extracts, $20 \mu \mathrm{g} / \mathrm{lane}$ ) were separated by SDS-PAGE, transferred to polyvinylidene fluoride (PVDF) membranes (Millipore) for $1 \mathrm{~h}$ at semi-dry, and blocked in blocking buffer for $1 \mathrm{~h}$ at room temperature. The PVDF membranes were incubated with the primary antibody against OCT-2 (1:1000 dilution), COX-2 ( $1: 1000$ dilution), cleaved caspase-3 (1:1000 dilution), and GAPDH ( $1: 1000$ dilution) overnight at $4{ }^{\circ} \mathrm{C}$, washed three times for $5 \mathrm{~min}$ in wash buffer, incubated with the HRP-conjugated secondary antibody ( $1: 2000$ dilution, anti-rabbit) for $1 \mathrm{~h}$ at room temperature, washed three times, and then detected with enhanced chemiluminescence (ECL) solution (GE Healthcare).

\section{Statistical analysis}

Statistical significance was determined through one-way analysis of variance (ANOVA) followed by a multiple comparison test with a Bonferroni adjustment. P values less than 0.05 were considered statistically significant. 


\section{Acknowledgements}

$\nabla$

This research was funded by the Gangneung Asan Hospital Biomedical Research Center Promotion Fund and Gachon University Research Fund 2014 (GCU-2014-0116).

\section{Conflict of Interest}

\section{$\nabla$}

The authors declare no conflict of interest.

\section{Affiliations}

${ }^{1}$ Department of Internal Medicine, University of Ulsan College of Medicine, Gangneung Asan Hospital, Gangneung, Korea

2 College of Korean Medicine, Gachon University, Seongnam, Korea

${ }^{3}$ Natural Products Research Institute, Korea Institute of Science and Technology, Gangneung, Korea

${ }^{4}$ Department of Food Science, Gyeongnam National University of Science and Technology, Jinju, Korea

${ }^{5}$ Department of Surgery, University of Ulsan College of Medicine, Gangneung Asan Hospital, Gangneung, Korea

${ }^{6}$ Laboratory of Molecular Oncology, Cheil General Hospital \& Women's Healthcare Center, Kwandong University College of Medicine, Seoul, Korea

\section{References}

1 Cai XZ, Huang WY, Qiao Y, Du SY, Chen Y, Chen D, Yu S, Che RC, Liu N, Jiang $Y$. Inhibitory effects of curcumin on gastric cancer cells: a proteomic study of molecular targets. Phytomedicine 2013; 20: 495-505

2 Wilken R, Veena MS, Wang MB, Srivatsan ES. Curcumin: a review of anti-cancer properties and therapeutic activity in head and neck squamous cell carcinoma. Mol Cancer 2011; 10: 12

3 Hurley LH. DNA and its associated processes as targets for cancer therapy. Nat Rev Cancer 2002; 2: 188-200

4 Miltenburg NC, Boogerd W. Chemotherapy-induced neuropathy: A comprehensive survey. Cancer Treat Rev 2014; 40: 872-882

5 Chorawala MR, Oza PM, Shah GB. Mechanisms of anticancer drugs resistance: an overview. Int J Pharm Sci Drug Res 2012; 4: 1-9

6 Arany I, Safirstein RL. Cisplatin nephrotoxicity. Semin Nephrol 2003; 23: 23460-23464

7 Zhu M, Chen J, Yin H, Jiang H, Wen M, Miao C. Propofol protects human umbilical vein endothelial cells from cisplatin-induced injury. Vascul Pharmacol 2014; 61: 72-79

8 Kohn S, Fradis M, Ben-David J, Zidan J, Robinson E. Nephrotoxicity of combined treatment with cisplatin and gentamicin in the guinea pig: glomerular injury findings. Ultrastruct Pathol 2002; 26: 371-382

9 Li Q Tian Y, Li D, Sun J, Shi D, Lin F, Gao Y, Liu H. The effect of lipocisplatin on cisplatin efficacy and nephrotoxicity in malignant breast cancer treatment. Biomaterials 2014; 35: 6462-6472

10 Luke DR, Vadiei K, Lopez-Berestein G. Role of vascular congestion in cisplatin induced acute renal failure in the rat. Nephrol Dial Transplant 1992; 7: 1-7

11 Miller RP, Tadagavadi RK, Ramesh G, Reeves WB. Mechanisms of Cisplatin nephrotoxicity. Toxins (Basel) 2010; 2: 2490-2518

12 Kang KS, Ham J, Kim YJ, Park JH, Cho EJ, Yamabe N. Heat-processed Panax ginseng and diabetic renal damage: active components and action mechanism. J Ginseng Res 2013; 37: 379-388

$13 \mathrm{Kim}$ JH, Han IH, Yamabe N, Kim YJ, Lee W, Eom DW, Choi P, Cheon GJ, Jang HJ, Kim SN, Ham J, Kang KS. Renoprotective effects of Maillard reaction products generated during heat treatment of ginsenoside Re with leucine. Food Chem 2014; 15: 114-121

14 Lee JH, Lee W, Lee S, Jung Y, Park SH, Choi P, Kim SN, Ham J, Kang KS. Important role of Maillard reaction in the protective effect of heat-processed ginsenoside Re-serine mixture against cisplatin-induced nephrotoxicity in LLC-PK1 cells. Bioorg Med Chem Lett 2012; 22: 54755479
15 Nath KA, Norby SM. Reactive oxygen species and acute renal failure. Am J Med 2000; 109: 665-678

16 Taguchi T, Nazneen A, Abid MR, Razzaque MS. Cisplatin-associated nephrotoxicity and pathological events. Contrib Nephrol 2005; 148: 107-121

17 Jiang $M$, Dong Z. Regulation and pathological role of p53 in cisplatin nephrotoxicity. Pharmacology 2008; 327: 300-307

18 Pabla N, Dong Z. Cisplatin nephrotoxicity: mechanisms and renoprotective strategies. Kidney Int 2008; 73: 994-1007

19 Yamabe N, Song KI, Lee W, Han IH, Lee JH, Ham J, Kim SN, Park JH, Kang $K S$. Chemical and free radical-scavenging activity changes of ginsenoside Re by Maillard reaction and its possible use as a renoprotective agent. J Ginseng Res 2012; 36: 256-262

20 Yamabe N, Kim YJ, Lee SY, Cho EJ, Park SH, Ham JY, Kim HY, Kang KS. Increase in antioxidant and anticancer effects of ginsenoside Re-lysine mixture by Maillard reaction. Food Chem 2013; 138: 876-883

21 Huang MT, Lysz T, Ferraro T, Abidi TF, Laskin JD, Conney AH. Inhibitory effects of curcumin on in vitro lipoxygenase and cyclooxygenase activities in mouse epidermis. Cancer Res 1991; 51: 813-819

22 Huang MT, Ma W, Yen P, Xie JG, Han J, Frenkel K, Grunberger D, Conney $A H$. Inhibitory effects of topical application of low doses of curcumin on 12-0-tetradecanoylphorbol-13-acetate-induced tumor promotion and oxidized DNA bases in mouse epidermis. Carcinogenesis 1997; 18: 83-88

23 Sandur SK, Ichikawa H, Pandey MK, Kunnumakkara AB, Sung B, Sethi G, Aggarwal BB. Role of pro-oxidants and antioxidants in the anti-inflammatory and apoptotic effects of curcumin (diferuloylmethane). Free Radic Biol Med 2007; 43: 568-580

24 Yoysungnoen P, Wirachwong P, Changtam C, Suksamrarn A, Patumraj S. Anti-cancer and anti-angiogenic effects of curcumin and tetrahydrocurcumin on implanted hepatocellular carcinoma in nude mice. World J Gastroenterol 2008; 7: 2003-2009

25 Murugan P, Pari L. Effect of tetrahydrocurcumin on lipid peroxidation and lipids in streptozotocin-nicotinamide-induced diabetic rats. Basic Clin Pharmacol Toxicol 2006; 99: 122-127

26 Yokozawa T, Cho EJ, Hara Y, Kitani K. Antioxidative activity of green tea treated with radical initiator 2, 2'-azobis (2-amidinopropane) dihydrochloride. J Agric Food Chem 2000; 48: 5068-5073

27 Forbes JM, Coughlan MT, Cooper ME. Oxidative stress as a major culprit in kidney disease in diabetes. Diabetes 2008; 57: 1446-1454

28 Heyman SN, Rosen S, Rosenberger C. A role for oxidative stress. Contrib Nephrol 2011; 174: 138-148

29 Li JM, Shah AM. ROS generation by nonphagocytic NADPH oxidase: potential relevance in diabetic nephropathy. J Am Soc Nephrol 2003; 14: 221-226

30 Takeda H, Sadakane C, Hattori T, Katsurada T, Ohkawara T, Nagai K, Asaka M. Rikkunshito, an herbal medicine, suppresses cisplatin-induced anorexia in rats via 5-HT2 receptor antagonism. Gastroenterology 2008; 134: 2004-2013

31 Jonker JW, Schinkel AH. Pharmacological and physiological functions of the polyspecific organic cation transporters: OCT1, 2, and 3 (SLC22A13). J Pharmacol Exp Ther 2004; 308: 2-9

32 Harris RC. Cyclooxygenase-2 in the kidney. J Cardiovasc Pharmacol 2006; 47: 37-42

$33 \mathrm{Kim}$ GH. Renal effects of prostaglandins and cyclooxygenase-2 inhibitors. Electrolyte Blood Press 2008; 6: 35-41

34 Cohen GM. Caspases: the executioners of apoptosis. Biochem 1997; 326: $1-16$

35 Yang B, Johnson TS, Thomas GL, Watson PF, Wagner B, Nahas AM. Apoptosis and caspase- 3 in experimental anti-glomerular basement membrane nephritis. J Am Soc Nephrol 2001; 12: 485-495

36 Mukhopadhyay P, Rajesh M, Pan H, Patel V, Mukhopadhyay B, Bátkai S, Gao B, Haskó G, Pacher P. Cannabinoid-2 receptor limits inflammation, oxidative/nitrosative stress, and cell death in nephropathy. Free Radic Biol Med 2010; 48: 457-467 